\title{
Heart Failure and Chronic Kidney Disease in Type 2 Diabetes
}

\author{
Simona Cernea \\ Department M3/Internal Medicine IV, University of Medicine and Pharmacy, Tîrgu Mureş, Romania \\ Diabetes, Nutrition and Metabolic Diseases Outpatient Unit, County Emergency Clinical Hospital, Tîrgu Mureş, Romania
}

\section{CORRESPONDENCE}

\section{Simona Cernea}

Str. Gheorghe Marinescu nr. 38

540139 Tîrgu Mureș, Romania

Tel: +40 265215551

E-mail: simona.cernea@umftgm.ro

\section{ARTICLE HISTORY}

Received: 9 December, 2016

Accepted: 22 December, 2016

\begin{abstract}
Complex hemodynamic, neurohormonal and biochemical changes occur in heart failure and chronic kidney disease, and hyperglycemia/diabetes further accentuate the multifactorial pathogenetic mechanisms. The acknowledgement of concomitant heart and kidney dysfunction in patients with type 2 diabetes has major clinical implications with regards to prognosis, as they significantly increase the risk of mortality, and to therapeutical strategy of both conditions, as well as of hyperglycemia. A comprehensive interdisciplinary approach is needed in these cases in order to improve the outcomes.
\end{abstract}

Keywords: type 2 diabetes, heart failure, chronic kidney disease, cardiorenal syndrome

\section{INTRODUCTION}

Heart failure (HF) and chronic kidney disease (CKD) coexist both in subjects with and without diabetes and are major health problems associated with significantly higher risk of mortality. ${ }^{1,2}$ Roughly, about a third to half of patients with $\mathrm{HF}$ present different degrees of $\mathrm{CKD}$, and the risk of death increases progressively with the severity of kidney dysfunction, the hazard ratio being more than 2 in patients with severe renal impairment. ${ }^{1,2}$ It seems that these two diseases are bidirectionally linked, and that the cardio-renal interaction is aggravated when diabetes superimposes. ${ }^{3}$

This is significant also in the context of the increasing prevalence of type 2 diabetes (T2D) worldwide. The International Diabetes Federation reports that currently 415 million adults have diabetes, and by 2040 this number is estimated to rise to 642 million. ${ }^{4}$ In parallel, the prevalence of CKD is also increasing lately, and apparently about $50 \%$ of patients with T2D suffer of CDK, diabetes being the leading cause of end-stage renal disease. ${ }^{4,5} \mathrm{On}$ the other hand, the prevalence of HF in patients with T2D was generally estimated to be about $9.5-22 \%$, but recent reports indicate that up to about $27 \%$ of patients with T2D have previously unknown HF, most of them with preserved ejection fraction (EF). ${ }^{6,7}$ Other data indicate that up to $50 \%$ of T2D patients may develop HF. ${ }^{8,9}$ The rising preva- 
lence of $\mathrm{T} 2 \mathrm{D}$ in young individuals and the demographic transition with more adults surviving longer (due to economic progress, improved healthcare and lifestyle) accentuate the changes in the epidemiology of CKD and HF. ${ }^{5}$

\section{THE CARDIORENAL SYNDROME}

The interaction between CKD and HF is complex and includes common risk factors (diabetes and hypertension probably being the most significant), bidirectional pathophysiological and clinical effects of one disease on the other, as well as therapeutical challenges, which are even more complicated in patients with T2D. ${ }^{3}$ It is thought that an insult on one organ (e.g. renal cortex or myocardium) causes adaptive responses and feedback loops that can influence the other organ and aggravates disease progression. ${ }^{10}$ The acknowledgement of this heart-kidney reciprocal link led to the definition of the cardiorenal syndrome (CRS), a term that expresses the understanding that one organ (dysfunction) affects the other. ${ }^{11}$ CRS exists in both acute and chronic conditions and is in fact classified in five types, according to the primary affected organ and the type of failure (acute, chronic or secondary).,12 The fifth type is secondary CRS, defined by systemic conditions (including diabetes) that lead to simultaneous cardiac and renal damage/dysfunction. ${ }^{3,12}$

The diagnosis of CKD is given by the presence of kidney damage (structural and/or functional abnormalities demonstrated on urine, blood, imaging or pathological tests) and/or decreased glomerular filtration rate (GFR), which is also used to stage CKD. ${ }^{3,11} \mathrm{HF}$ is a clinical condition caused by an impairment of the cardiac pump function and defined by typical signs (most common, peripheral edema), symptoms (most typical, shortness of breath and fatigue) and objective evidence of structural/functional abnormalities (e.g. increased natriuretic peptide and echocardiographic changes). ${ }^{3}$ Both have several stages of progression during which they interact in a complex interdependent manner and create a vicious cycle that can accelerate the deterioration of both organs. Their interaction is mediated and accentuated by several mechanisms/factors, including hemodynamic, neurohormonal, cardiovascular and others (oxidative stress, inflammation, metabolic factors etc.), which will be further reviewed. ${ }^{11}$

\section{RISK FACTORS AND PATHOGENETIC MECHANISMS}

It is well known that diabetes is a causal factor for kidney damage, but whether it is also for HF or just a comorbidity, is less clear, although it seems that it promotes myocardial fibrosis and dysfunction. ${ }^{13}$ T2D is a complex disease with hyperglycemia at its core, resulting from many causes: insulin deficiency caused by reduction of $\beta$ cell function/ mass, insulin resistance, incretin deficiency, hyperglucagonemia, increased glucose reabsorption in the kidney, neurohormonal dysfunction. Diabetes is viewed as a traditional cardiovascular risk factor for heart and kidney disease, along with hypertension, obesity and dyslipidemia, but many other factors/mechanisms/molecules are also involved in the cardio-renal interaction. ${ }^{3,10}$

The key hemodynamic mechanisms include water and sodium retention with fluid overload, cardiac and renal congestion and decreased organ perfusion. ${ }^{11} \mathrm{HF}$ with preserved or decreased EF can be associated with decreased cardiac output, which induces renal feedback mechanisms in order to maintain renal plasma flow and glomerular filtration. ${ }^{11}$ The regulatory mechanisms include activation of the renin-angiotensin-aldosterone system (RAAS) and tubulo-glomerular feedback with changes in renal blood flow and perfusion pressures, renal vasoconstriction and sodium and water retention. ${ }^{11}$ It should be noted that several reports indicated that high right atrial pressure and central venous pressure (and not necessarily the low cardiac output) correlate with decreased GFR. ${ }^{11,14,15}$ These pressures are typically increased in $\mathrm{HF}$ and associated with renal venous hypertension, which in turn induces a decline in renal function through multiple mechanisms. ${ }^{11}$ Venous renal hypertension/renal congestion activates neurohormonal responses (such as sympathetic nervous system [SNS] and RAAS), inflammation and fibrogenesis, which further accentuates the neurohormonal and inflammatory responses. ${ }^{11}$

The main neurohormonal mechanisms consist in the activation of RAAS and SNS, both essential in CKD and HF. ${ }^{11}$ Increased activity of the RAAS determines water and sodium retention with intravascular volume expansion, vasoconstriction and increased end-diastolic ventricle pressure. ${ }^{11}$ Angiotensin II and aldosterone stimulate the release of natriuretic peptides (atrial natriuretic peptide and B-type natriuretic peptide, also released in response to other neurohormonal and mechanical stimuli, such as increased cardiac wall stress, which reduces peripheral vascular resistance, myocardial fibrosis and hypertrophy and reduces aldosterone secretion), arginine vasopressin (causing vasoconstriction, fluid retention and hyponatremia), pro-inflammatory cytokines, macrophage-derived galectin-3 (causing cardiac and renal fibrosis and remodeling, inflammation and oxidative stress). ${ }^{11,16,17}$ In HF, the natriuretic peptide system is not able to counterbalance 


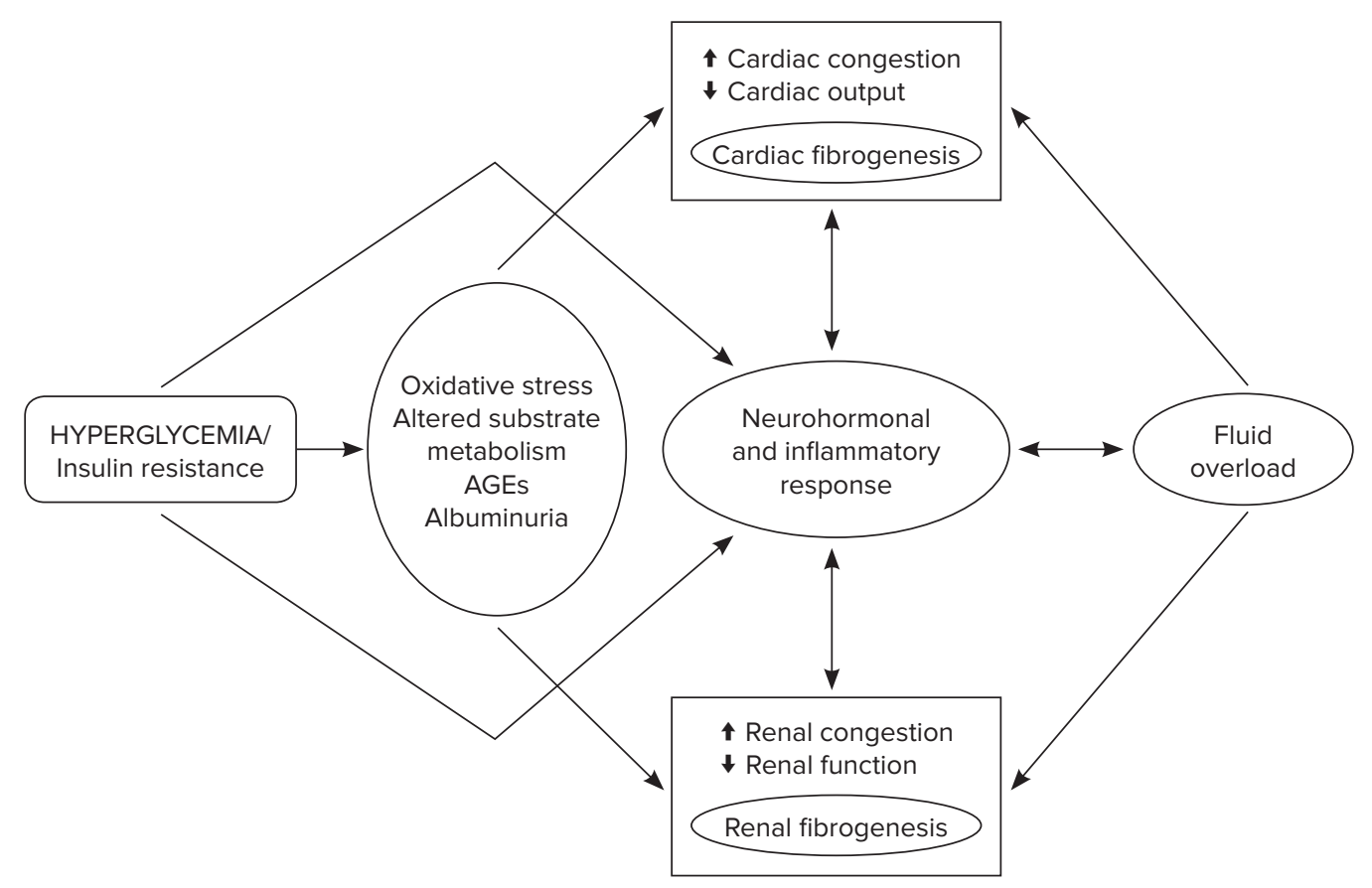

FIGURE 1. The main mechanisms of cardio-renal interaction in type 2 diabetes

the RAAS and sodium-retention it induces, therefore there is a fluid overload, increased venous return and cardiac congestion. ${ }^{11,18}$ This creates and accentuates a vicious cycle that aggravates the progression of the disease (Figure 1). SNS stimulation also activates the RAAS and promotes inflammation and apoptosis. ${ }^{19}$

A number of other mechanisms are possibly involved, such as chronic inflammation, anemia, metabolic changes (e.g. bone mineral disorders) or cardiovascular disease and comorbidities (atherosclerosis, hypertension, diabetes etc.). ${ }^{11}$ There is a large body of literature indicating that chronic inflammation and associated changes in the cellular immune system (activation of $\mathrm{T}$ lymphocytes, antigen- presenting cells) are important players in the promotion and progression of HF and CKD. ${ }^{11,19}$ Many factors can contribute to the induction of chronic inflammation, such as SNS activation, oxidative stress, fluid retention with venous congestion, uremic toxins, obesity, diabetes, nutritional, environmental and genetic factors etc., and it is documented by the increase of biomarkers (C-reactive protein, pro-inflammatory cytokines, pentraxin-3 etc.). ${ }^{11,18-20}$ Increased inflammation and oxidative stress lead to fibrosis and remodeling in both organs, as well as endothelial dysfunction. ${ }^{11,19,21}$ High levels of reactive oxygen species (ROS) induce several pathways/ molecules, such as transforming growth factor (TGF)- $\beta$, nuclear factor (NF)-kB and galectin-3, which are involved in inflammation and interstitial fibrosis though upregu- lation and proliferation of fibroblasts and production of procollagen. ${ }^{19,21}$

Anemia and bone-mineral axis are also important players in accelerating the progression of CKD and HF. ${ }^{11,22}$ Patients with CKD have decreased ability to synthesize erythropoietin and have disrupted iron metabolism. ${ }^{22}$ On the other hand, a considerable proportion (up to a third) of patients with HF present anemia. ${ }^{23,24}$ Persistent anemia may be involved in ventricular hypertrophy/dilatation, impairment of myocytes' mechanical function and exacerbation of HF, as well as activation of RAAS, renal vasoconstriction and diminished renal function. ${ }^{22}$ Thus, it is not only a biomarker, but a contributor to disease progression. ${ }^{11}$ In addition, bone mineral disorders with defective metabolism of vitamin $\mathrm{D}$, parathyroid hormone (PTH), phosphate, calcium and fibroblast growth factor (FGF) 23 contribute to the CRS, by promoting cardiac hypertrophy and fibrosis, vascular stiffness and calcification, endothelial cell toxicity, and decreased renal functional mass. ${ }^{22}$

\section{The role of diabetes/hyperglycemia in heart and kidney dysfunction}

Finally, a key role in the pathogenesis and progression of CRS in patients with T2D is played by hyperglycemia and glucotoxicity through direct and indirect mechanisms, including the alternative biochemical cascades in which excessive glucose is diverted and which contribute to 
the hyperglycemia-induced cellular injury and apoptosis (Figure 1).25

The mechanisms by which T2D and hyperglycemia induce renal damage are extensively studied. The formation of advanced glycation end-products (AGEs), the increase of cytokines/inflammation and growth factor levels, oxidative and hemodynamic stress with increased endothelial permeability/dysfunction are associated with mesangial proliferation, renal sclerosis/fibrosis and proteinuria and are known to be the main mechanisms of kidney dysfunction. ${ }^{12}$ Activated TGF $\beta$ and vascular endothelial growth factor (VEGF) are important players in diabetic nephropathy and CKD by inducing the thickening of glomerular basement membrane and podocyte structural changes/ loss with glomerulosclerosis. ${ }^{12,26}$ Hyperglycemia, and possibly insulin resistance, induce through effector pathways endothelial dysfunction, which leads to simultaneous cardiac and renal impairment. ${ }^{12,27}$ High blood glucose levels can upregulate the RAAS by increasing tissue angiotensin and aldosterone that induce all the pathogenetic mechanisms mentioned above. ${ }^{28}$ In addition, hyperglycemia reduces the nitric oxide (NO) bioavailability and thus impairs vasodilation and contributes to vascular complications in T2D. ${ }^{12}$

Inflammatory cytokines, ROS and growth factors induced by hyperglycemia are also important contributors to the pathogenesis of HF in T2D. The AGEs generated extra- and intracellularly cross-link proteins and remodel the extracellular matrix, the pro-inflammatory cytokines and chemokines recruit fibrogenic leukocytes in the myocardial interstitial tissue and cause endothelial dysfunction, while ROS transduces fibrogenic signals and induces ultrastructural changes (e.g. myofibrillary degeneration). ${ }^{29}$ The activation of cardiac fibrosis decreases myocardial compliance/increases stiffness, induces changes in ventricular geometry and may even trigger arrhythmic events. ${ }^{29,30}$ Reports also indicated that in diabetic cardiomyopathy there is an impaired calcium handling (possibly due to non-enzymatic glycation) and dysfunction/abnormalities in mitochondrial bioenergetics/structure (possibly caused by oxidative stress), which reduce systolic and diastolic function. ${ }^{25,30} \mathrm{~T} 2 \mathrm{D}$ is associated with dysregulation of the RAAS, and angiotensin II acts not only on cardiomyocytes, but on insulin-sensitive tissues as well, where it affects the insulin receptor, insulin receptor substrates and signaling pathways (e.g. phosphatidylinositol-3-kinase [PI3K]/protein kinase B [PKB], glucose transporters [GLUT] 4) and reduces insulin sensitivity. ${ }^{25}$ Thus, the dysregulation of the RAAS is not only involved in the pathogenesis of HF and kidney disease, but in insulin resistance as well. Insulin re- sistance in cardiomyocytes is also accentuated by the Fox (Forkhead box) $\mathrm{O}$ transcription factors by interfering with the insulin signaling pathways (PI3K/PKB). ${ }^{25}$

Hyperglycemia and insulin resistance are associated with altered substrate metabolism in the myocardium. Hyperlipidemia and lipotoxicity with the accumulation of toxic lipid intermediates impair calcium homeostasis and cardiomyocyte contraction. ${ }^{25,30}$ They also lead to increased oxygen demands, generation of ROS and ceramide production, which may trigger apoptosis. ${ }^{25,30}$ Thus, hyperglycemia is associated with alterations of myocyte metabolism, cardiac ultrastructure and endothelial function. ${ }^{6}$ In addition, hyperinsulinemia/insulin resistance seen in patients with T2D and obesity may be associated with a cross-talk between signaling pathways and stimulation of the cascades involved in protein synthesis and cell growth. ${ }^{25}$ All these have structural and functional consequences, and several typical changes are seen in the diabetic heart: left ventricular hypertrophy, diastolic dysfunction and systolic dysfunction (generally observed in more advanced stages). ${ }^{30}$

In addition, it appears that adipokines such as leptin, resistin and adiponectin also play a role in the CRS in T2D. ${ }^{25,31-33}$ Part of the effects are mediated by receptors and post-receptor activating mechanisms, e.g. adenosine monophosphate-activated protein kinase (AMPK) and mitogen-activated protein kinase (MAPK), and possibly $\mathrm{Ca}^{2+}$ metabolism. ${ }^{31-33}$ Adipokines can contribute to the CRS through hemodynamic effects (e.g. leptin), endothelial dysfunction, oxidative stress, inflammation and SNS. ${ }^{31-34}$

It should be mentioned that patients with $\mathrm{T} 2 \mathrm{D}$ associate hypertension and obesity in high proportion, and these two comorbidities are also involved in mechanisms linked to renal dysfunction and HF, which further amplify the pathogenetic mechanisms and disease progression. Obesity imposes hemodynamic changes that concomitantly affects the heart (diastolic dysfunction) and kidney (decreased GFR). ${ }^{35}$ Thus, the inter-relation between T2D and its comorbidities (obesity, hypertension) on one hand and CRS on the other, is complex.

\section{THERAPEUTIC ASPECTS}

The therapeutic recommendations in patients with T2D and CRS rely on those specific for each of the diseases. ${ }^{11}$ The goals are to improve clinical status, functional capacity, quality of life and reduce hospitalization and mortality. ${ }^{36}$

In general, several recommendations are made: avoidance of nephrotoxic and cardiotoxic agents, lifestyle optimization, lipid and blood pressure control, drug therapy for fluid/hemodynamic control etc. ${ }^{11}$ The reduction of 
caloric and alcohol intake and sustained physical exercise, mainly at the early stages of cardio-renal dysfunction have important benefits by ameliorating systemic and tissue insulin resistance and hemodynamic disturbances. ${ }^{31}$ In T2D patients with non-dialyzed CKD the recommended protein intake is $0.8 \mathrm{~g} / \mathrm{kg} /$ day, which slows GFR decline. ${ }^{9}$ Weight loss (even by ways of bariatric surgery) is associated with favorable metabolic changes and attenuates CRS by improving organ perfusion. ${ }^{37}$

The ESC guidelines recommend angiotensin-converting enzyme (ACE) inhibitors (or angiotensin II receptor blockers [ARBs]), $\beta$ blockers, mineralocorticoid/aldosterone-receptor blockers and diuretics for the treatment of HF. ${ }^{36}$ ACE inhibitors and $\beta$ blockers are considered firstline therapy in all symptomatic HF patients with reduced $\mathrm{EF}$ and were proven to reduce mortality and morbidity in these patients. ${ }^{36}$ Diuretics are used to reduce congestion in HF patients and they seem to improve exercise capacity. ${ }^{36}$ ACE inhibitors/ARBs and diuretics are also used in diabetic nephropathy/CKD. ${ }^{9}$ ACE inhibitors/ARBs are the preferred first-line agents for the treatment of hypertension in $\mathrm{T} 2 \mathrm{D}$ and were shown to reduce the progression of CKD and albuminuria. ${ }^{9}$

Blood glucose control is at the core of T2D management. Studies have shown that intensive glycemic control reduces microvascular complications, while the macrovascular benefits are variable: on long-term it seems to confer cardiovascular protection, mainly if started early in the disease course, but an aggressive glucose-lowering approach in subjects with long-standing T2D and advanced cardiovascular disease may inflict harm. ${ }^{12,38}$ Thus, the management of patients with T2D should be done in an individualized manner, considering not only the glycemic targets, but also the presence and severity of comorbidities, as well as the cardiovascular safety of glucose-lowering drugs. 38

At present, several classes of antihyperglycemic drugs are available for the treatment of T2D. Some of these agents have cardiac/renal protective effects, others may have some detrimental effects or are contraindicated in HF or CKD (or need dose adjustments/precaution). Thus, the presence of $\mathrm{HF}$ and/or CKD complicates the treatment of T2D.

Metformin is the preferred initial antihyperglycemic drug for the treatment of T2D. ${ }^{9}$ It improves insulin resistance and may even exert some cardioprotective effects through the activation of AMPK, suppression of inflammatory responses and inhibition of aldosterone-induced cardiac fibrosis, and may reduce the development of HF. ${ }^{25,39-41}$ On the other hand, it is contraindicated if GFR falls below $30 \mathrm{~mL} / \mathrm{min} / 1.73 \mathrm{~m}^{2} .{ }^{9}$
Thiazolidindiones (TZDs) are associated with increased risk of $\mathrm{HF}$ in patients with T2D and therefore should be avoided in those with symptomatic HF. ${ }^{9,42}$ Patients treated with TZDs should be carefully monitored for signs and symptoms of HF, especially after therapy start-up and at each dose escalation. ${ }^{38}$ However, since pioglitazone is metabolized in the liver, no dose adjustment in necessary in patients with renal impairment. ${ }^{43}$ In patients with more advanced CKD, sulphonylureas should be avoided due to decreased renal clearance and higher risk of hypoglycemia, which may be associated with unwanted cardiovascular outcomes. ${ }^{44}$

There are several newer therapeutic classes for the treatment of T2D, such as glucagon-like peptide 1 (GLP-1) receptor agonists, dipeptidyl peptidase 4 (DPP-4) inhibitors and sodium-glucose cotransporter-2 (SGLT2) inhibitors, and a number of large outcome trials have evaluated their cardiovascular safety.

Data in the literature indicated that the GLP-1 receptor agonists may have some cardioprotective benefits. ${ }^{25}$ The Evaluation of Lixisenatide in Acute Coronary Syndrome (ELIXA) trial demonstrated non-inferiority of lixisenatide (a short-acting GLP-1 receptor agonist) vs. placebo for cardiovascular outcomes, including hospitalization for HF. ${ }^{45}$ However, the Liraglutide and Cardiovascular Outcomes in Type 2 Diabetes (LEADER) trial showed cardiovascular benefits (primary composite endpoint) of liraglutide vs. placebo, including on cardiovascular mortality. ${ }^{46}$ However, no benefits for hospitalization for HF were observed with the longer-acting GLP-1 receptor agonist liraglutide and interestingly, major cardiovascular events were reduced to a greater degree in patients with impaired renal function (GFR $<60 \mathrm{~mL} / \mathrm{min} / 1.73 \mathrm{~m}^{2}$ ). ${ }^{44,46} \mathrm{GLP}-1$ receptor agonists are contraindicated in CKD stages 4 and 5 , and some need dose adjustment in stage $3 .{ }^{43}$ The renoprotective effects of this class were not clearly proven yet, although there are some data suggesting a reduction in albuminuria. ${ }^{47}$

The three outcome clinical trials that evaluated the cardiovascular safety of DPP-4 inhibitors - the Saxagliptin Assessment of Vascular Outcomes Recorded in Patients with Diabetes Mellitus-Thrombolysis in Myocardial Infarction 53 (SAVOR-TIMI53), the Examination of Cardiovascular Outcomes with Alogliptin vs. Standard of Care (EXAMINE) and the Trial Evaluating Cardiovascular Outcomes with Sitagliptin (TECOS) - showed cardiovascular safety as they all proved non-inferiority vs. placebo with regards to the primary endpoint. ${ }^{48-50}$ However, the HF findings were conflicting, with unexpected and concerning results regarding the increased risk of hospitalization for HF with saxagliptin vs. placebo that was not explained 
so far, while the other two members of the class were not associated with higher risk of HF hospitalization..$^{38,48-51} \mathrm{On}$ the other hand, saxagliptin reduced progressive albuminuria regardless of baseline renal function. ${ }^{52}$ The potential renoprotective effect (reduction of albuminuria) was seen with the other DPP-4 inhibitors as well. ${ }^{43}$ They all can be used in all stages of CKD with dose adjustments, with the exception of linagliptin, which can be used without reduction of dose. ${ }^{43}$

Finally, the (Empagliflozin) Cardiovascular Outcome Event Trial in Type 2 Diabetes Mellitus Patients (EMPAREG OUTCOME) study demonstrated that empaglifozin, a SGLT2 inhibitor, was associated with clear cardiovascular benefits with regards to the primary composite endpoint, cardiovascular mortality and reduced risk of hospitalization for HF (35\%). ${ }^{53}$ The results of other cardiovascular outcome trials with SGLT2 inhibitors are still waited. The mechanisms of cardiovascular and renal protective effects are not entirely clear, but it is thought that they go beyond the improvement of glycemia or cardiovascular risk factors (decrease in blood pressure and plasma volume, increased uricosuria, reduction of total body and epicardial fat, inflammation and fibrosis) and improved tubulo-glomerular feedback mechanisms. ${ }^{54}$ It was suggested that changes in substrate and fuel energetics are involved, which improve the transduction of oxygen consumption into work efficiency in heart and kidney mitochondria, but further studies are needed. ${ }^{55}$ SGLT2 inhibitors are contraindicated or require dose adjustment in patients with CKD stage 3-5.43

Given all these, and since there is relatively limited evidence (dedicated clinical trials) of specific therapeutic approach of patients with T2D and concomitant HF and CKD, the most appropriate conduct would be a multidisciplinary approach with continuous interactions between specialists. The best treatment assumes an individualized approach according to underlying pathophysiological changes. ${ }^{56}$

\section{CONCLUSIONS}

Complex hemodynamic, neurohormonal, biochemical changes are involved in $\mathrm{CKD}$ and $\mathrm{HF}$, and diabetes further accentuates the pathogenetic mechanisms. When these two conditions are concomitantly present in patients with $\mathrm{T} 2 \mathrm{D}$, there is a particularly high risk of poor health outcomes. Therefore, a timely and appropriate evaluation of heart and kidney function is needed. The care of patients with CKD and HF is challenging, especially when T2D is also present. Furthermore, the management of diabetes per se is complicated and needs special precaution in choosing antihyperglycemic agents and adjusting their dosage.

\section{CONFLICT OF INTEREST}

Nothing to declare.

\section{REFERENCES}

1. Damman K, Valente MA, Voors AA, et al. Renal impairment, worsening renal function, and outcome in patients with heart failure: an updated meta-analysis. Eur Heart J. 2014;35:455-469

2. Löfman I, Szummer K, Hagerman I, et al. Prevalence and prognostic impact of kidney disease on heart failure patients. Open Heart. 2016;3:e000324.

3. Shiba N, Shimokawa H. Chronic kidney disease and heart failure Bidirectional close link and common therapeutic goal. J Cardiol. 2011;57:8-17.

4. International Diabetes Federation. IDF Diabetes Atlas, 2015. Available at: http://www.diabetesatlas.org

5. Thomas MC, Cooper ME, Zimmet P. Changing epidemiology of type 2 diabetes mellitus and associated chronic kidney disease. Nat Rev Nephrol. 2016;12:73-81.

6. Roberts AW, Clark AL, Witte KK. Review article: Left ventricular dysfunction and heart failure in metabolic syndrome and diabetes without overt coronary artery disease — do we need to screen our patients? Diab Vasc Dis Res. 2009;6:153-163.

7. Boonman-de Winter LJ, Rutten FH, Cramer MJ, et al. High prevalence of previously unknown heart failure and left ventricular dysfunction in patients with type 2 diabetes. Diabetologia. 2012;55:2154-2162.

8. Kannel WB, Hjortland M, Castelli WP. Role of diabetes in congestive heart failure: the Framingham study. Am J Cardiol. 1974;34:29-34.

9. American Diabetes Association. Standards of Medical Care in Diabetes-2017. Diabetes Care. 2017;40(Suppl1):S1-S135.

10. Re RN. A Reassessment of the Pathophysiology of Progressive Cardiorenal Disorders. Med Clin North Am. 2017;101:103-115.

11. Schefold JC, Filippatos G, Hasenfuss G, et al. Heart failure and kidney dysfunction: epidemiology, mechanisms and management. Nat Rev Nephrol. 2016;12:610-623.

12. Karnib $\mathrm{HH}$, Ziyadeh FN. The cardiorenal syndrome in diabetes mellitus Diabetes Res Clin Pract. 2010;89:201-208.

13. McMurray JJ, Gerstein HC, Holman RR, Pfeffer MA. Heart failure: a cardiovascular outcome in diabetes that can no longer be ignored. Lancet Diabetes Endocrinol. 2014;2:843-851.

14. Mullens W, Abrahams Z, Francis GS, et al. Importance of venous congestion for worsening of renal function in advanced decompensated heart failure. J Am Coll Cardiol. 2009;53:589-596.

15. Damman K, van Deursen VM, Navis G, Voors AA, van Veldhuisen DJ, Hillege HL. Increased central venous pressure is associated with impaired renal function and mortality in a broad spectrum of patients with cardiovascular disease. J Am Coll Cardiol. 2009;53:582-588.

16. Ramos HR, Birkenfeld AL, de Bold AJ. INTERACTING DISCIPLINES: Cardiac natriuretic peptides and obesity: perspectives from an endocrinologist and a cardiologist. Endocr Connect. 2015;4:R25-R36.

17. Farmakis D, Filippatos G, Kremastinos DT, Gheorghiade M. Vasopressin and vasopressin antagonists in heart failure and hyponatremia. Curr Heart Fail Rep. 2008;5:91-96.

18. Lekawanvijit S, Krum H. Cardiorenal syndrome: acute kidney injury secondary to cardiovascular disease and role of protein-bound uraemic toxins. J Physiol. 2014;592:3969-3983.

19. Rubattu S, Mennuni S, Testa M, et al. Pathogenesis of chronic cardiorenal syndrome: is there a role for oxidative stress? Int J Mo/ Sci. 2013;14:2301123032.

20. Ma RC. Genetics of cardiovascular and renal complications in diabetes. $J$ Diabetes Investig. 2016;7:139-154.

21. Hundae A, McCullough PA. Cardiac and renal fibrosis in chronic cardiorenal syndromes. Nephron Clin Pract. 2014;127:106-112.

22. Charytan DM, Fishbane S, Malyszko J, McCullough PA, Goldsmith D. Cardiorenal Syndrome and the Role of the Bone-Mineral Axis and Anemia. Am J Kidney Dis. 2015;66:196-120.

23. Groenveld HF, Januzzi JL, Damman K, et al. Anemia and mortality in heart failure patients a systematic review and meta-analysis. J Am Coll Cardiol. 2008;52:818-827. 
24. Young JB, Abraham WT, Albert NM, et al. Relation of low hemoglobin and anemia to morbidity and mortality in patients hospitalized with heart failure (insight from the OPTIMIZE-HF registry). Am J Cardiol. 2008;101:223-230.

25. Battiprolu PK, Gillette TG, Wang ZV, Lavandero S, Hill JA. Diabetic Cardiomyopathy: Mechanisms and Therapeutic Targets. Drug Discov Today Dis Mech. 2010;7:e135-e143

26. Garud MS, Kulkarni YA. Hyperglycemia to nephropathy via transforming growth factor beta. Curr Diabetes Rev. 2014;10:182-189.

27. Satchell SC, Tooke JE. What is the mechanism of microalbuminuria in diabetes: a role for the glomerular endothelium? Diabetologia 2008;51:714-725

28. Giacchetti G, Sechi LA, Rilli S, Carey RM. The renin-angiotensinaldosterone system, glucose metabolism and diabetes. Trends Endocrinol Metab. 2005;16:120-126.

29. Russo I, Frangogiannis NG. Diabetes-associated cardiac fibrosis: Cellular effectors, molecular mechanisms and therapeutic opportunities. J Mol Cell Cardiol. 2016:90:84-93.

30. Yilmaz S, Canpolat U, Aydogdu S, Abboud HE. Diabetic Cardiomyopathy Summary of 41 Years. Korean Circ J. 2015;45:266-272.

31. Aroor AR, Mandavia $\mathrm{CH}$, Sowers JR. Insulin resistance and heart failure: molecular mechanisms. Heart Fail Clin. 2012;8:609-617.

32. Sweiss N, Sharma K. Adiponectin effects on the kidney. Best Pract Res Clin Endocrinol Metab. 2014;28:71-79.

33. Nasrallah MP, Ziyadeh FN. Overview of the physiology and pathophysiology of leptin with special emphasis on its role in the kidney. Semin Nephrol. 2013:33:54-65.

34. Rüster C1, Wolf G. Adipokines promote chronic kidney disease. Nephrol Dial Transplant. 2013;28(Suppl4):iv8-iv14.

35. Ybarra J, Sánchez-Hernández J, Vilallonga R, Romeo JH. Differentia associations between glomerular filtration rate and duration of obesity depending on the presence or absence of left ventricular diastolic dysfunction. Eur J Intern Med. 2016;32:47-52.

36. Ponikowski P, Voors AA, Anker SD, et al. 2016 ESC Guidelines for the diagnosis and treatment of acute and chronic heart failure: The Task Force for the diagnosis and treatment of acute and chronic heart failure of the European Society of Cardiology (ESC)Developed with the special contribution of the Heart Failure Association (HFA) of the ESC. Eur J Heart Fail. 2016;18:891-975.

37. Fenske W, Athanasiou T, Harling L, Drechsler C, Darzi A, Ashrafian H. Obesity-related cardiorenal disease: the benefits of bariatric surgery. Nat Rev Nephrol. 2013;9:539-551.

38. Cahn A, Cernea S, Raz I. Outcome studies and safety as guide for decision making in treating patients with type 2 diabetes. Rev Endocr Metab Disord. 2016:17:117-127.

39. Mummidi S, Das NA, Carpenter AJ, et al. Metformin inhibits aldosteroneinduced cardiac fibroblast activation, migration and proliferation in vitro, and reverses aldosterone+salt-induced cardiac fibrosis in vivo. J Mol Cell Cardiol. 2016:98:95-102.
40. Soraya H, Clanachan AS, Rameshrad M, Maleki-Dizaji N, Ghazi-Khansari M, Garjani A. Chronic treatment with metformin suppresses toll-like receptor 4 signaling and attenuates left ventricular dysfunction following myocardial infarction. Eur J Pharmacol. 2014;737:77-84.

41. El Messaoudi S, Rongen GA, Riksen NP. Metformin therapy in diabetes: the role of cardioprotection. Curr Atheroscler Rep. 2013;15:314

42. Singh S, Loke YK, Furberg CD. Thiazolidinediones and heart failure: teleo-analysis. Diabetes Care. 2007;30:2148-2153.

43. Davies M, Chatterjee S, Khunti K. The treatment of type 2 diabetes in the presence of renal impairment: what we should know about newer therapies. Clin Pharmacol. 2016;8:61-81.

44. Aguilar D. Heart Failure, Diabetes Mellitus, and Chronic Kidney Disease: A Clinical Conundrum. Circ Heart Fail. 2016;97pii:e003316.

45. Pfeffer MA, Claggett B, Diaz R, et al. ELIXA Investigators. Lixisenatide in Patients with Type 2 Diabetes and Acute Coronary Syndrome. N Engl J Med. 2015:373:2247-2257.

46. Marso SP, Daniels GH, Brown-Frandsen K, et al. Liraglutide and Cardiovascular Outcomes in Type 2 Diabetes. N Engl J Med. 2016;375:311 322

47. von Scholten BJ, Hansen TW, Goetze JP, Persson F, Rossing P. Glucagonlike peptide 1 receptor agonist (GLP-1 RA): long-term effect on kidney function in patients with type 2 diabetes. J Diabetes Complications. 2015;29:670-674

48. Scirica BM, Bhatt DL, Braunwald E, et al. Saxagliptin and cardiovascular outcomes in patients with type 2 diabetes mellitus. N Engl J Med. 2013;369:1317-1326

49. Zannad F, Cannon CP, Cushman WC, et al. Heart failure and mortality outcomes in patients with type 2 diabetes taking alogliptin versus placebo in EXAMINE: a multicentre, randomised, double-blind trial. Lancet. 2015;385:2067-2076

50. Green JB, Bethel MA, Armstrong PW, et al. Effect of Sitagliptin on Cardiovascular Outcomes in Type 2 Diabetes. N Engl J Med. 2015;373:232 242.

51. Cernea S, Cahn A, Raz I. Saxagliptin for the treatment of diabetes - a focus on safety. Expert Opin Drug Saf. 2016;15:697-707.

52. Udell JA, Bhatt DL, Braunwald E, et al. Saxagliptin and cardiovascular outcomes in patients with type 2 diabetes and moderate or severe rena impairment: observations from the SAVOR-TIMI 53 Trial. Diabetes Care. 2015;38:696-705

53. Zinman B, Wanner C, Lachin JM, et al. Empagliflozin, Cardiovascular Outcomes, and Mortality in Type 2 Diabetes. N Engl J Med. 2015;373:21172128.

54. Rajasekeran H, Lytvyn Y, Cherney DZ. Sodium-glucose cotransporter 2 inhibition and cardiovascular risk reduction in patients with type 2 diabetes: the emerging role of natriuresis. Kidney Int. 2016;89:524-526.

55. Ferrannini E, Mark M, Mayoux E. CV Protection in the EMPA-REG OUTCOME Trial: A "Thrifty Substrate" Hypothesis. Diabetes Care. 2016;39:1108-1114.

56. Carubelli V, Lombardi C, Gorga E, Ravera A, Metra M, Mentz RJ. Cardiorenal Interactions. Heart Fail Clin. 2016:12:335-347. 